\title{
THE EFFECT OF POROUS MEDIUM ON PULSATILE INCLINED TWO LAYERED BLOOD FLOW WITH MAGNETIC FIELD AND PERIODIC BODY ACCELERATION
}

\author{
Varun Kumar Tanwar ${ }^{1}$, Dr. N. K.Varshney ${ }^{2}$, Raja Agarwal ${ }^{3}$ \\ 1,2Deptt. of Mathematics, S.V.College, Aligarh, \\ ${ }^{3}$ Deptt. of Mathematics, Accurate Institute of Management and Technology, Gr.Noida,
}

\begin{abstract}
A mathematical model has been developed for studying the effect of porous medium on pulsatile inclined two layered blood flow with magnetic field with periodic body acceleration.Considering the Blood as couple stresses, non-Newtonian, and incompressible fluid. Finite Hankel and Laplace transform are used to analytical expression for the velocity profile and flow rate. The effect of various parameters associated with this flow problem such as porosity, Hartmann number, pressure gradient, body acceleration, gravitational parameter and inclination angle on the velocity distribution is analysed by plotting the graph. It is found that velocity decreases with the increase of the Hartmann number and the reverse behavior is noticed for the pressure gradient and body acceleration. It is also observed that the velocity profile increases with the increase of the permeability of the porous medium.
\end{abstract}

Keywords: Pulsatile blood flow, Permeability of porous medium, Magnetic field, Body acceleration.

\section{Introduction}

Many cardiovascular diseases, particularly atherosclerosis, have been found to be responsible for deaths in both developed and developing countries. Blood is considered to be one of the most important multicomponent mixtures. It is composed of plasma, red cells and white cells (RBCs and WBCs), platelets, etc. The study of porous medium with magnetic field is very important both from theoretical as well as practical point of view; because most of natural phenomena of the fluid flow are connected with porous medium. For instance, filtration of fluids, under- ground water and oil, reservoir and fluid through pipes.

Various mathematical models have been investigated by several researchers to explore the behaviour of blood flow under the influence of external magnetic field. Agarwal and Varshney ${ }^{21}$ studied the effect of magnetic field of pulsatile inclined two layered blood flow with periodic body acceleration .Agarwal and Varshney ${ }^{19}$ studied inclined arterial MHD pulsatile blood flow through a porous medium with periodic body acceleration. Amos and Ogulu ${ }^{13}$ discussed magnetic effect on pulsatile flow in a constricted axisymmetric tube. Astarita et.al ${ }^{1}$ investigated non-Newtonian gravity flow on inclined surface. Chaturani and Palanisamy ${ }^{5}$ considered Casson fluid model for pulsatile flow of blood under periodic body acceleration. Chaturani and Palanisamy ${ }^{6}$ studied pulsatile flow of Power-law fluid model for blood flow under periodic body acceleration. Chaturani and Wassf Isaac ${ }^{9}$ discussed blood flow with body acceleration forces. El-Shehed ${ }^{14}$ considered $^{1}$ pulsatile flow of blood through a stenosed porous medium under periodic body acceleration. Haldar and Ghosh ${ }^{8}$ discussed effect of magnetic field on blood flow through an indented tube in the presence of erythrocytes. Kavitha and Hemadri Reddy et.al ${ }^{18}$ studied Peristaltic flow of a micropolar fluid in a vertical channel with long wave length approximation. Rathod and Gopichand ${ }^{15}$ considered pulsatile flow of blood through a stenosed inclined tube under periodic body acceleration. Rathod and Ahmed ${ }^{10}$ investigated pulsatile two layered flow under periodic body acceleration. Rathod and Channakote ${ }^{11}$ studied a study ureteral peristalsis in cylindrical tube through porous medium. Rathod and HosurkerShrikanth ${ }^{12}$ considered MHD flow of Rivlin-Ericksen fluid through an inclined channel. Ravi Kumar et. $\mathrm{Al}^{17}$ considered peristaltic transport of a power-law fluid in an asymmetric channel bounded by permeable walls. Sapna Singh and Rajeev Ratan Shah ${ }^{15}$ studied a numerical model for the effect of stenosis shape on blood flow through an artery using power-law fluid. Srivastava and Saxena ${ }^{7}$ investigated two-layered model of Casson's fluid flow through stenotic blood vessels application to cardiovascular system. Krishna Kumari and, Ramana Murthy et.al ${ }^{20}$ considered Peristaltic pumping of a magnetohydrodynamic casson fluid in an inclined channel.Stokes ${ }^{2}$ studied couple-stress in fluid. Sud and Sekhon ${ }^{4}$ considered blood flow through the human arterial system in the presence of a steady magnetic field. Sud et.al ${ }^{3}$ discussed effect of magnetic field on oscillating blood in arteries. Tranter ${ }^{22}$ studied integral transform in mathematical physics, infinite transform.

In the present section we have considered the problem of Agarwal and Varshney [1] introducing porous medium under the same conditions taken by Agarwal and Varshney[1]. 


\section{Mathematical Formulation}

Let us consider a one-dimensional pulsatile inclined two layer flow of blood with periodic permeability of porous medium in the influence of external magnetic field by considering blood as a couple stresses, nonNewtonian and incompressible fluid. Consider the flow as axially symmetric, pulsatile and fully developed. The pressure gradient and body acceleration $\mathrm{G}$ are given by:

$$
\begin{array}{cc}
-\frac{\partial P}{\partial z}=A_{0}+A_{1} \cos \left(\omega_{p} t\right), & t \geq 0, \\
G=a_{0} \cos \left(\omega_{b} t+\phi\right), & t \geq 0,
\end{array}
$$

Where $A_{0}$ and $A_{1}$ are pressure gradient of steady flow and amplitude of oscillatory part respectively, $a_{0}$ is the amplitude of body acceleration, $\omega_{p}=2 \pi f_{p}, \omega_{b}=2 \pi f_{b}$ with $f_{p}$ is the pulse frequency and $f_{b}$ is body acceleration frequency, $\phi$ is the phase angle of body acceleration $G$ with respect to the pressure gradient and $t$ is time.

The governing equation of motion for flow in cylindrical polar coordinates

$$
\begin{gathered}
\rho_{1} \frac{\partial u_{1}}{\partial t}=-\frac{\partial P}{\partial z}+\rho_{1} G+\mu_{1} \nabla^{2} u_{1}-\eta \nabla^{2}\left(\nabla^{2} u_{1}\right)+\rho_{1} g \sin \theta-\sigma B_{0}^{2} u_{1}-\frac{\mu_{1}}{K} u_{1} \\
\rho_{2} \frac{\partial u_{2}}{\partial t}=-\frac{\partial P}{\partial z}+\rho_{2} G+\mu_{2} \nabla^{2} u_{2}+\rho_{2} g \sin \theta \\
\text { Where } \nabla^{2}=\frac{1}{r}\left(\frac{\partial}{\partial r}\left(r \frac{\partial}{\partial r}\right)\right)
\end{gathered}
$$

Where $u_{i}(r)$ is velocity in the axial direction, $P$ is the blood pressure, $\frac{\partial P}{\partial z}$ is the pressure gradient, $\rho_{1}$ is the density of blood, $\mu_{1}$ is the viscosity of blood, $\rho_{2}$ is the density of plasma, $\mu_{2}$ is the viscosity of plasma, $B_{0}$ is the external magnetic field along the radial direction and $\sigma$ is the electrical conductivity, $r$ is the radial coordinate and $\eta$ is the couple stress parameter, $g$ is acceleration due to gravity, $\theta$ is the inclined angle. $K$ is the permeability of porous medium.

Let us introduce the following dimensionless quantities:

$$
\begin{array}{ll}
u_{i}^{*}=\frac{u_{i}}{\omega R}, \quad r^{*}=\frac{r}{R}, \quad t^{*}=t \omega, & A_{0}^{*}=\frac{R}{\mu \omega} A_{0},
\end{array}
$$

Where $i=1,2$

In terms of these variables, equation (3) and (4) [after dropping stars] becomes

$$
\begin{aligned}
\bar{\alpha}^{2} \alpha^{2} \frac{\partial u_{1}}{\partial t}= & \bar{\alpha}^{2}\left(A_{0}+A_{1} \cos t+a_{0} \cos (b t+\phi)\right)+\bar{\alpha}^{2}\left(\frac{1}{r} \frac{\partial}{\partial r}\left(r \frac{\partial u_{1}}{\partial r}\right)\right) \\
& -\left(\frac{1}{r} \frac{\partial}{\partial r}\left(r \frac{\partial}{\partial r}\right)\right) \cdot\left(\frac{1}{r} \frac{\partial}{\partial r}\left(r \frac{\partial u_{1}}{\partial r}\right)\right)-\bar{\alpha}^{2} M+\bar{\alpha}^{2} g \sin \theta
\end{aligned}
$$




$$
\alpha^{2} \frac{\partial u_{2}}{\partial t}=A_{0}+A_{1} \cos t+a_{0} \cos (b t+\phi)+g \sin \theta+\left(\frac{1}{r} \frac{\partial}{\partial r}\left(r \frac{\partial u_{2}}{\partial r}\right)\right)
$$

Where

$$
\bar{\alpha}^{2}=\frac{R^{2} \mu}{\eta} \text { is couple stress parameter, } \alpha=R\left(\frac{\omega \rho}{\mu}\right)^{\frac{1}{2}} \text { is Womersley parameter, }
$$

$M_{1}=H^{2}+\frac{1}{K}, H=B_{0} R\left(\frac{\sigma}{\mu}\right)^{\frac{1}{2}}$ is the Hartmann number, $b=\frac{\omega_{b}}{\omega_{p}}$ and $R$ is the radius of the tube.

The initial and boundary conditions for the problem are

$$
u_{1}(r, 0)=\frac{\frac{2}{h} \sum_{n=1}^{\infty} \frac{\lambda_{n} \bar{\alpha}^{2}}{\left(s^{2}+\lambda_{n}^{2}\right)}\left[A_{0}+A_{1}+a_{0} \cos \phi+g \sin \theta\right] J_{1}\left(h \lambda_{n}\right)}{\left\{\left[\lambda_{n}^{4}+\bar{\alpha}^{2}\left(\lambda_{n}^{2}+M_{1}\right)\right]\right.} \times \frac{J_{0}\left(r \lambda_{n}\right)}{J_{0}^{2}\left(r \lambda_{n}\right)}
$$

$$
u_{2}(r, 0)=\frac{\pi^{2}}{2} \sum_{n=1}^{\infty} \frac{\lambda_{n}^{2} J_{0}^{2}\left(\lambda_{n}\right)\left\{\begin{array}{l}
h \lambda_{n} u_{2}(h) B_{0}^{\prime}\left(h \lambda_{n}\right)+\frac{1}{\lambda_{n}}\left[A_{0}+A_{1}\right. \\
\left.+a_{0} \cos \phi+g \sin \theta\right] \times\left[h B_{0}^{\prime}\left(h \lambda_{n}\right)-B_{0}^{\prime}\left(\lambda_{n}\right)\right]
\end{array}\right\}}{\left\{J_{0}^{2}\left(h \lambda_{n}\right)-J_{0}^{2}\left(\lambda_{n}\right)\right\} \lambda_{n}^{2}} \times B_{0}\left(r \lambda_{n}\right)
$$

Where

$$
\begin{gathered}
u_{2}(h)=\frac{\frac{2}{h} \sum_{n=1}^{\infty} \frac{\lambda_{n} \bar{\alpha}^{2}}{\left(s^{2}+\lambda_{n}^{2}\right)}\left[A_{0}+A_{1}+a_{0} \cos \phi+g \sin \theta\right] J_{1}\left(h \lambda_{n}\right)}{\left\{\begin{array}{l}
{\left[\lambda_{n}^{4}+\bar{\alpha}^{2}\left(\lambda_{n}^{2}+M_{1}\right)\right]} \\
\left.-\frac{2 \lambda_{n}^{2}}{h^{2}\left(s^{2}+\lambda_{n}^{2}\right)}\left[\frac{2}{h^{2}}-\frac{\lambda_{n} J_{1}\left(h \lambda_{n}\right)}{h J_{0}\left(h \lambda_{n}\right)}+h\left(\lambda_{n}^{2}+\bar{\alpha}^{2}+\frac{M_{1}}{\lambda_{n}^{2}}\right) \frac{\lambda_{n} J_{1}\left(h \lambda_{n}\right)}{J_{0}\left(h \lambda_{n}\right)}\right]\right\}
\end{array}\right] \frac{1}{J_{0}\left(h \lambda_{n}\right)}} \\
u_{1} \text { and } \nabla^{2} u_{1} \text { are all finite } \quad \text { at } r=0 \\
u_{1}=u_{2} \text { and } \frac{\partial u_{2}}{\partial r}=0 \quad \text { at } r=h \\
u_{2}=0 \text { and } \nabla^{2} u_{2}=0 \quad \text { at } r=1
\end{gathered}
$$




\section{Required Integral Transforms}

The finite Hankel transform, defined by Tranter [22], over the interval $(0, \mathrm{~h})$ is

$$
u^{*}\left(\lambda_{n}, t\right)=\int_{0}^{h} r u J_{n}\left(r \lambda_{n}\right) d r
$$

and its inverse transform is $u(r, t)=\frac{2}{h^{2}} \sum_{n=1}^{\infty} u^{*}\left(\lambda_{n}\right) \frac{J_{n}\left(r \lambda_{n}\right) \lambda_{n}^{2}}{J_{n}^{2}\left(h \lambda_{n}\right)\left(s^{2}+\lambda_{n}^{2}\right)}$

The Hankel transform over the interval (h, 1), defined by Tranter [22],

$$
u^{*}\left(\lambda_{n}, t\right)=\int_{h}^{1} r u B_{n}\left(r \lambda_{n}\right) d r
$$

and its appropriate inverse transform is

$$
u(r, t)=\frac{\pi^{2}}{2} \sum_{n=1}^{\infty} u^{*}\left(\lambda_{n}\right) \frac{J_{n}^{2}\left(\lambda_{n}\right) \lambda_{n}^{2}}{J_{n}^{2}\left(h \lambda_{n}\right)-J_{n}^{2}\left(\lambda_{n}\right)} u^{*}\left(\lambda_{n}\right) B_{n}\left(r \lambda_{n}\right)
$$

Where

$$
B_{n}\left(\lambda_{n} r\right)=J_{n}\left(\lambda_{n} r\right) Y\left(\lambda_{n} h\right)-Y\left(\lambda_{n} r\right) J_{n}\left(\lambda_{n} h\right)
$$

$\lambda_{n}$ are positive roots of the equation $J_{n}\left(\lambda_{n}\right) Y_{n}\left(\lambda_{n} h\right)-Y_{n}\left(\lambda_{n}\right) J_{n}\left(\lambda_{n} h\right)=0$

and $J_{n}$ and $Y_{n}$ are the Bessel's functions of first and second kind of order $n$ respectively.

The Laplace transform of any function is defined as:

$$
\bar{f}(s)=\int_{0}^{\infty} e^{-s t} f(t) d t, \quad s>0
$$

\section{Solution Of The Problem}

Applying Laplace transform (17) to equation (6) in the light of $(8,10,11)$ we get

$$
\begin{aligned}
\bar{\alpha}^{2} \alpha^{2}\left(s \bar{u}_{1}-u_{1}(r, 0)\right) & =\bar{\alpha}^{2} \frac{\left(A_{0}+g \sin \theta\right)}{s}+\bar{\alpha}^{2} \frac{A_{1} s}{\left(s^{2}+1\right)}+\bar{\alpha}^{2} \frac{a_{0}(s \cos \phi-b \sin \phi)}{\left(s^{2}+b^{2}\right)} \\
& +\bar{\alpha}^{2}\left(\frac{1}{r} \frac{\partial}{\partial r}\left(r \frac{\partial \bar{u}_{1}}{\partial r}\right)\right)-\left(\frac{1}{r} \frac{\partial}{\partial r}\left(r \frac{\partial}{\partial r}\right)\right) \cdot\left(\frac{1}{r} \frac{\partial}{\partial r}\left(r \frac{\partial \bar{u}_{1}}{\partial r}\right)\right) \\
& -\bar{\alpha}^{2} M_{1} \bar{u}_{1}
\end{aligned}
$$

Next applying the finite Hankel transform (13) to (18) which gives as 


$$
\begin{gathered}
\bar{u}_{1}^{*}=\left\{\begin{array}{c}
\frac{h J_{1}\left(h \lambda_{n}\right) \bar{\alpha}^{2}}{\lambda_{n}}\left[\frac{A_{0}+g \sin \theta}{s}+\frac{A_{1} s}{\left(s^{2}+1\right)}+\frac{a_{0}(s \cos \phi-b \sin \phi)}{\left(s^{2}+b^{2}\right)}\right] \\
+\left[h \lambda_{n} J_{1}\left(h \lambda_{n}\right)\left(\bar{\alpha}^{2}+\lambda_{n}^{2}+\frac{\bar{\alpha}^{2} M_{1}}{\lambda_{n}^{2}}\right)+\frac{2}{h^{2}} J_{0}\left(h \lambda_{n}\right)-\frac{\lambda_{n} J_{1}\left(h \lambda_{n}\right)}{h}\right] \bar{u}_{2}+m x
\end{array}\right\} \\
\times \frac{1}{\left[s m+\left\{\lambda_{n}^{4}+\bar{\alpha}^{2}\left(\lambda_{n}^{2}+M_{1}\right)\right\}\right]}
\end{gathered}
$$

Now rearranging the terms of (19) to take inverse Laplace transform

$$
\begin{aligned}
& \bar{u}_{1}^{*}=\left\{\frac { h J _ { 1 } ( h \lambda _ { n } ) \overline { \alpha } ^ { 2 } } { \lambda _ { n } } \left[\frac{A_{0}+g \sin \theta}{\left\{\lambda_{n}^{4}+\bar{\alpha}^{2}\left(\lambda_{n}^{2}+M_{1}\right)\right\}}\left(\frac{1}{s}-\frac{1}{s+h_{1}}\right)\right.\right. \\
& +\frac{A_{1}\left[\lambda_{n}^{4}+\bar{\alpha}^{2}\left(\lambda_{n}^{2}+M_{1}\right)\right]}{\left[\lambda_{n}^{4}+\bar{\alpha}^{2}\left(\lambda_{n}^{2}+m^{2}+M_{1}\right)\right]}\left(\frac{s}{s^{2}+1}-\frac{1}{s+h_{1}}+\frac{m}{\left(s^{2}+1\right)\left[\lambda_{n}^{4}+\bar{\alpha}^{2}\left(\lambda_{n}^{2}+M_{1}\right)\right]}\right) \\
& +\frac{a_{0}\left[\lambda_{n}^{4}+\bar{\alpha}^{2}\left(\lambda_{n}^{2}+M_{1}\right)\right] \cos \phi}{\left[\left\{\lambda_{n}^{4}+\bar{\alpha}^{2}\left(\lambda_{n}^{2}+M_{1}\right)\right\}^{2}+m^{2} b^{2}\right]}\left(\frac{s}{s^{2}+b^{2}}-\frac{1}{s+h_{1}}\right) \\
& -\frac{a_{0} m b \sin \phi}{\left.\left(s^{2}+b^{2}\right)\left[\lambda_{n}^{4}+\bar{\alpha}^{2}\left(\lambda_{n}^{2}+M_{1}\right)\right]\right)} \\
& \left.+\left\{\lambda_{n}^{4}+\bar{\alpha}^{2}\left(\lambda_{n}^{2}+M_{1}\right)\right\}^{2}+m^{2} b^{2}\right]\left(-\frac{s}{s^{2}+b^{2}}+\frac{1}{s+h_{1}}+\frac{\left[\lambda_{n}^{4}+\bar{\alpha}^{2}\left(\lambda_{n}^{2}+M_{1}\right)\right]}{\left(s^{2}+b^{2}\right) m}\right) \\
& +\left[h \lambda_{n} J_{1}\left(h \lambda_{n}\right)\left(\bar{\alpha}^{2}+\lambda_{n}^{2}+\frac{\bar{\alpha}^{2} M_{1}}{\lambda_{n}^{2}}\right)+\frac{2 J_{0}\left(h \lambda_{n}\right)}{h^{2}}-\frac{\lambda_{n} J_{1}\left(h \lambda_{n}\right)}{h}\right] \bar{u}_{2} \\
& \left.\times \frac{1}{m}\left(\frac{1}{s+h_{1}}\right)+\left(\frac{1}{s+h_{1}}\right) x\right\}
\end{aligned}
$$

Now taking inverse Laplace transform to (20) and rearranging the term we get 


$$
\begin{aligned}
& u_{1}^{*}=\left\{\frac { h J _ { 1 } ( h \lambda _ { n } ) \overline { \alpha } ^ { 2 } } { \lambda _ { n } } \left[\frac{A_{0}+g \sin \theta}{\left\{\lambda_{n}^{4}+\bar{\alpha}^{2}\left(\lambda_{n}^{2}+M_{1}\right)\right\}}+\frac{A_{1}\left[\left\{\lambda_{n}^{4}+\bar{\alpha}^{2}\left(\lambda_{n}^{2}+h^{2}+M_{1}\right)\right\} \cos t+m \sin t\right]}{\left[\left\{\lambda_{n}^{4}+\bar{\alpha}^{2}\left(\lambda_{n}^{2}+h^{2}+M_{1}\right)\right\}^{2}+m^{2}\right]}\right.\right. \\
&\left.+\frac{a_{0}\left[\left\{\lambda_{n}^{4}+\bar{\alpha}^{2}\left(\lambda_{n}^{2}+h^{2}+M_{1}\right)\right\} \cos (b t+\phi)+m b \sin (b t+\phi)\right]}{\left[\left\{\lambda_{n}^{4}+\bar{\alpha}^{2}\left(\lambda_{n}^{2}+h^{2}+M_{1}\right)\right\}^{2}+m^{2} b^{2}\right]}\right] \\
&-e^{-h_{1} t}\left[\frac { h J _ { 1 } ( h \lambda _ { n } ) \overline { \alpha } ^ { 2 } } { \lambda _ { n } } \left\{\frac{A_{0}+g \sin \theta}{\left[\lambda_{n}^{4}+\bar{\alpha}^{2}\left(\lambda_{n}^{2}+h^{2}+M_{1}\right)\right]}+\frac{A_{1}\left[\lambda_{n}^{4}+\bar{\alpha}^{2}\left(\lambda_{n}^{2}+h^{2}+M_{1}\right)\right]}{\left.\left[\lambda_{n}^{4}+\bar{\alpha}^{2}\left(\lambda_{n}^{2}+h^{2}+M_{1}\right)\right\}^{2}+m^{2}\right]}\right.\right. \\
&+\frac{a_{0}\left[\left\{\lambda_{n}^{4}+\bar{\alpha}^{2}\left(\lambda_{n}^{2}+h^{2}+M_{1}\right)\right\} \cos \phi+m b \sin \phi\right]}{\left[\left\{\lambda_{n}^{4}+\bar{\alpha}^{2}\left(\lambda_{n}^{2}+h^{2}+M_{1}\right)\right\}^{2}+m^{2} b^{2}\right]}+\left[h \lambda_{n} J_{1}\left(h \lambda_{n}\right)\left(\bar{\alpha}^{2}+\lambda_{n}^{2}+\frac{\bar{\alpha}^{2} M_{1}}{\lambda_{n}^{2}}\right)\right. \\
&\left.\left.\left.+\frac{2 J_{0}\left(h \lambda_{n}\right)}{h^{2}}-\frac{\lambda_{n} J_{1}\left(h \lambda_{n}\right)}{h}\right] \frac{u_{2}}{m}+x\right]\right\}
\end{aligned}
$$

Now taking the finite Hankel inversion (14) gives the final solution as:

$$
\begin{aligned}
u_{1}(r, t)=\frac{2}{h^{2}} \sum_{n=1}^{\infty} & \frac{J_{0}\left(r \lambda_{n}\right) \lambda_{n}^{2}}{J_{0}^{2}\left(h \lambda_{n}\right)\left(s^{2}+\lambda_{n}^{2}\right)}\left\{\frac { h J _ { 1 } ( h \lambda _ { n } ) \overline { \alpha } ^ { 2 } } { \lambda _ { n } } \left[\frac{A_{0}+g \sin \theta}{\left\{\lambda_{n}^{4}+\bar{\alpha}^{2}\left(\lambda_{n}^{2}+M_{1}\right)\right\}}\right.\right. \\
& +\frac{A_{1}\left[\left\{\lambda_{n}^{4}+\bar{\alpha}^{2}\left(\lambda_{n}^{2}+h^{2}+M_{1}\right)\right\} \cos t+m \sin t\right]}{\left[\left\{\lambda_{n}^{4}+\bar{\alpha}^{2}\left(\lambda_{n}^{2}+h^{2}+M_{1}\right)\right\}^{2}+m^{2}\right]} \\
& \left.+\frac{a_{0}\left[\left\{\lambda_{n}^{4}+\bar{\alpha}^{2}\left(\lambda_{n}^{2}+h^{2}+M_{1}\right)\right\} \cos (b t+\phi)+m b \sin (b t+\phi)\right]}{\left[\left\{\lambda_{n}^{4}+\bar{\alpha}^{2}\left(\lambda_{n}^{2}+h^{2}+M_{1}\right)\right\}^{2}+m^{2} b^{2}\right]}\right] \\
& -e^{-h_{1} t}\left[\frac { h _ { 1 } ( h \lambda _ { n } ) \overline { \alpha } ^ { 2 } } { \lambda _ { n } } \left\{\frac{A_{0}+g \sin \theta}{\left[\lambda_{n}^{4}+\bar{\alpha}^{2}\left(\lambda_{n}^{2}+h^{2}+M_{1}\right)\right]}+\left\{\lambda_{n}^{4}+\bar{\alpha}^{2}\left(\lambda_{n}^{2}+h^{2}+M_{1}\right)\right]\right.\right. \\
+ & \frac{a_{0}\left[\left\{\lambda_{n}^{4}+\bar{\alpha}^{2}\left(\lambda_{n}^{2}+h^{2}+M_{1}\right)\right\}^{2}+m^{2}\right]}{\left.\left[\left\{\lambda_{n}^{2}+h^{2}+M_{1}\right)\right\} \cos \phi+m b \sin \phi\right]}
\end{aligned}
$$




$$
\begin{aligned}
& +\frac{\frac{1}{m}\left[h \lambda_{n} J_{1}\left(h \lambda_{n}\right)\left(\bar{\alpha}^{2}+\lambda_{n}^{2}+\frac{\left.\bar{\alpha}^{2} M_{1}\right)}{\lambda_{n}^{2}}\right)+\frac{2 J_{0}\left(h \lambda_{n}\right)}{h^{2}}-\frac{\lambda_{n} J_{1}\left(h \lambda_{n}\right)}{h}\right]}{\left\{1+\frac{2 \lambda_{n}^{2}}{h^{2} J_{0}\left(h \lambda_{n}\right)\left(s^{2}+\lambda_{n}^{2}\right)}\left[h \lambda_{n} J_{1}\left(h \lambda_{n}\right)\left(\bar{\alpha}^{2}+\lambda_{n}^{2}+\frac{\bar{\alpha}^{2} M_{1}}{\lambda_{n}^{2}}\right)+\frac{2 J_{0}\left(h \lambda_{n}\right)}{h^{2}}-\frac{\lambda_{n} J_{1}\left(h \lambda_{n}\right)}{h}\right] \frac{1}{m}\right\}} \\
& \times \frac{2 \lambda_{n}^{2}}{h^{2} J_{0}\left(h \lambda_{n}\right)\left(s^{2}+\lambda_{n}^{2}\right)}\left\{\frac { h J _ { 1 } ( h \lambda _ { n } ) \overline { \alpha } ^ { 2 } } { \lambda _ { n } } \left[\frac{A_{0}+g \sin \theta}{\left\{\lambda_{n}^{4}+\bar{\alpha}^{2}\left(\lambda_{n}^{2}+M_{1}\right)\right\}}\right.\right. \\
& +\frac{A_{1}\left[\left\{\lambda_{n}^{4}+\bar{\alpha}^{2}\left(\lambda_{n}^{2}+h^{2}+M_{1}\right)\right\} \cos t+m \sin t\right]}{\left[\left\{\lambda_{n}^{4}+\bar{\alpha}^{2}\left(\lambda_{n}^{2}+h^{2}+M_{1}\right)\right\}^{2}+m^{2}\right]} \\
& \left.+\frac{a_{0}\left[\left\{\lambda_{n}^{4}+\bar{\alpha}^{2}\left(\lambda_{n}^{2}+h^{2}+M_{1}\right)\right\} \cos (b t+\phi)+m b \sin (b t+\phi)\right]}{\left[\left\{\lambda_{n}^{4}+\bar{\alpha}^{2}\left(\lambda_{n}^{2}+h^{2}+M_{1}\right)\right\}^{2}+m^{2} b^{2}\right]}\right] \\
& -e^{-h_{1} t}\left[\frac { h J _ { 1 } ( h \lambda _ { n } ) \overline { \alpha } ^ { 2 } } { \lambda _ { n } } \left\{\frac{A_{0}+g \sin \theta}{\left[\lambda_{n}^{4}+\bar{\alpha}^{2}\left(\lambda_{n}^{2}+h^{2}+M_{1}\right)\right]}+\frac{A_{1}\left[\lambda_{n}^{4}+\bar{\alpha}^{2}\left(\lambda_{n}^{2}+h^{2}+M_{1}\right)\right]}{\left[\left\{\lambda_{n}^{4}+\bar{\alpha}^{2}\left(\lambda_{n}^{2}+h^{2}+M_{1}\right)\right\}^{2}+m^{2}\right]}\right.\right. \\
& \left.\left.\left.\left.\left.\left.+\frac{a_{0}\left[\left\{\lambda_{n}^{4}+\bar{\alpha}^{2}\left(\lambda_{n}^{2}+h^{2}+M_{1}\right)\right\} \cos \phi+m b \sin \phi\right]}{\left[\left\{\lambda_{n}^{4}+\bar{\alpha}^{2}\left(\lambda_{n}^{2}+h^{2}+M_{1}\right)\right\}^{2}+m^{2} b^{2}\right]}\right\}+x\right]\right\}+x\right\}\right]\right\}
\end{aligned}
$$

Where $m=\bar{\alpha}^{2} \alpha^{2}, h_{1}=\frac{\left[\lambda_{n}^{4}+\bar{\alpha}^{2}\left(\lambda_{n}^{2}+h^{2}+M_{1}\right)\right]}{m}$

$$
x=\frac{h \sum_{n=1}^{\infty} \frac{\lambda_{n} \bar{\alpha}^{2}}{\left(s^{2}+\lambda_{n}^{2}\right)}\left[A_{0}+A_{1}+a_{0} \cos \phi+g \sin \theta\right] J_{1}\left(h \lambda_{n}\right)}{\left\{\begin{array}{r}
{\left[\lambda_{n}^{4}+\bar{\alpha}^{2}\left(\lambda_{n}^{2}+M_{1}\right)\right]} \\
\left.-\frac{2 \lambda_{n}^{2}}{h^{2}\left(s^{2}+\lambda_{n}^{2}\right)}\left[\frac{2}{h^{2}}-\frac{\lambda_{n} J_{1}\left(h \lambda_{n}\right)}{h J_{0}\left(h \lambda_{n}\right)}+h\left(\lambda_{n}^{2}+\bar{\alpha}^{2}+\frac{M_{1}}{\lambda_{n}^{2}}\right) \frac{\lambda_{n} J_{1}\left(h \lambda_{n}\right)}{J_{0}\left(h \lambda_{n}\right)}\right]\right\} \\
\times \frac{J_{0}^{2}\left(h \lambda_{n}\right)+J_{1}^{2}\left(h \lambda_{n}\right)}{J_{0}^{2}\left(h \lambda_{n}\right)}
\end{array}\right.}
$$


Next applying the Laplace transform (17) to equation (7) using of (9), (10), (11), and (12), we obtained,

$$
\begin{aligned}
\alpha^{2}\left(s \bar{u}_{2}-u_{2}(r, 0)\right)=\frac{\left(A_{0}+g \sin \theta\right)}{s}+\frac{A_{1} s}{\left(s^{2}+1\right)}+\frac{a_{0}(s \cos \phi-b \sin \phi)}{\left(s^{2}+b^{2}\right)} \\
+\left(\frac{1}{r} \frac{\partial}{\partial r}\left(r \frac{\partial \bar{u}_{2}}{\partial r}\right)\right)
\end{aligned}
$$

Now applying the finite Hankel transform (13) to (23) and rearranging the term as

$$
\begin{gathered}
\bar{u}_{2}^{*}=\left[\frac{A_{0}+g \sin \theta}{\lambda_{n}^{2}}\left(\frac{1}{s}-\frac{1}{s+h_{2}}\right)+\frac{A_{1} \lambda_{n}^{2}}{\left(\lambda_{n}^{2}+\alpha^{2}\right)}\left(\frac{s}{s^{2}+1}-\frac{1}{s+h_{2}}+\frac{\alpha^{2}}{\left(s^{2}+1\right) \lambda_{n}^{2}}\right)\right. \\
+\frac{a_{0} \lambda_{n}^{2} \cos \phi}{\left[\lambda_{n}^{2}+\alpha^{2} b^{2}\right]}\left(\frac{s}{s^{2}+b^{2}}-\frac{1}{s+h_{2}}+\frac{\alpha b^{2}}{\left(s^{2}+b^{2}\right) \lambda_{n}^{2}}\right) \\
\left.-\frac{a_{0} \alpha b \sin \phi}{\left[\lambda_{n}^{4}+\alpha^{2} b^{2}\right]}\left(-\frac{s}{s^{2}+b^{2}}-\frac{1}{s+h_{2}}+\frac{\lambda_{n}^{2}}{\left(s^{2}+b^{2}\right) \alpha}\right)\right] \\
\times \frac{1}{\lambda_{n}}\left[h B_{0}^{\prime}\left(h \lambda_{n}\right)-B_{0}^{\prime}\left(h \lambda_{n}\right)\right] \\
+\left[h \lambda_{n} u_{2}(h, t) B_{0}^{\prime}\left(h \lambda_{n}\right)\right] \frac{1}{\alpha^{2}}\left(\frac{1}{s+h_{2}}\right)+Y Z \frac{1}{\alpha^{2}}\left(\frac{1}{s+h_{2}}\right)
\end{gathered}
$$

Now taking inverse Laplace transform to (24) and rearranging the terms finite inverse Hankel transform as,

$$
\begin{aligned}
& u_{2}^{*}=\left[\frac{A_{0}+g \sin \theta}{\lambda_{n}^{2}}+\frac{A_{1}\left[\lambda_{n}^{2} \cos t+\alpha^{2} \sin t\right]}{\left(\lambda_{n}^{4}+\alpha^{4}\right)}\right. \\
&\left.+\frac{a_{0}\left[\lambda_{n}^{2} \cos (b t+\phi)+\alpha^{2} b \sin (b t+\phi)\right]}{\left(\lambda_{n}^{4}+\alpha^{4} b^{2}\right)}\right] \frac{1}{\lambda_{n}}\left[h B_{0}^{\prime}\left(h \lambda_{n}\right)-B_{0}^{\prime}\left(h \lambda_{n}\right)\right] \\
&-e^{-h_{2} t}\left[\frac { [ h B _ { 0 } ^ { \prime } ( h \lambda _ { n } ) - B _ { 0 } ^ { \prime } ( \lambda _ { n } ) ] } { \lambda _ { n } } \left\{\frac{A_{0}+g \sin \theta}{\lambda_{n}^{2}}+\frac{A_{1} \lambda_{n}^{2}}{\left(\lambda_{n}^{4}+\alpha^{4} b^{2}\right)}\right.\right. \\
&\left.\left.\left.+\frac{a_{0}\left[\lambda_{n}^{2} \cos \phi+\alpha^{2} b \sin \phi\right]}{\left(\lambda_{n}^{4}+\alpha^{4} b^{2}\right)}\right\}-\frac{1}{\alpha^{2}}\left\{h \lambda_{n} u_{2}(h, t) B_{0}^{\prime}\left(h \lambda_{n}\right)\right\}-Y Z\right\}\right]
\end{aligned}
$$

Now taking the inverse Hankel transform (14) to (25), which gives final solution as, 


$$
\begin{aligned}
u_{2}(r, t) & =\frac{\pi^{2}}{2} \sum_{n=1}^{\infty} \frac{\lambda_{n}^{2} J_{0}^{2}\left(\lambda_{n}\right)}{J_{0}^{2}\left(h \lambda_{n}\right)-J_{0}^{2}\left(\lambda_{n}\right)} B_{0}\left(r \lambda_{n}\right)\left[\left\{X_{1} \frac{1}{\lambda_{n}}\left[h B_{0}^{\prime}\left(h \lambda_{n}\right)-B_{0}^{\prime}\left(\lambda_{n}\right)\right]\right\}\right. \\
& -e^{-h_{2} t}\left\{\left[X_{2} \frac{1}{\lambda_{n}}\left[h B_{0}^{\prime}\left(h \lambda_{n}\right)-B_{0}^{\prime}\left(\lambda_{n}\right)\right]\right]-\frac{1}{\alpha^{2}}\left[h B_{0}^{\prime}\left(h \lambda_{n}\right)\right]\right. \\
& \times \frac{\pi^{2}}{2} \sum_{n=1}^{\infty} \frac{\lambda_{n}^{2} J_{0}^{2}\left(\lambda_{n}\right)}{J_{0}^{2}\left(h \lambda_{n}\right)-J_{0}^{2}\left(\lambda_{n}\right)} B_{0}\left(h \lambda_{n}\right)\left\{X_{1} \frac{1}{\lambda_{n}}\left[h B_{0}^{\prime}\left(h \lambda_{n}\right)-B_{0}^{\prime}\left(\lambda_{n}\right)\right]\right. \\
& \left.-e^{-h_{2} t}\left[X_{2} \frac{1}{\lambda_{n}}\left\{h B_{0}^{\prime}\left(h \lambda_{n}\right)-B_{0}^{\prime}\left(\lambda_{n}\right)\right\}+\frac{Y Z}{\alpha^{2}}\right]\right\}
\end{aligned}
$$

$$
\begin{gathered}
\left.\left.\left.\times \frac{1}{\left[1-\frac{\lambda_{n}^{2} J_{0}^{2}\left(\lambda_{n}\right) B_{0}^{\prime}\left(h \lambda_{n}\right)}{J_{0}^{2}\left(h \lambda_{n}\right)-J_{0}^{2}\left(\lambda_{n}\right)} \frac{1}{\alpha^{2}}\left[h \lambda_{n} B_{0}^{\prime}\left(h \lambda_{n}\right) e^{-h_{2} t}\right]\right]}-Y Z\right]\right\}\right] \\
X_{1}=\left[\frac{A_{0}+g \sin \theta}{\lambda_{n}^{2}}+\frac{A_{1}\left[\lambda_{n}^{2} \cos t+\alpha^{2} \sin t\right]}{\left(\lambda_{n}^{4}+\alpha^{4}\right)}+\frac{a_{0}\left[\lambda_{n}^{2} \cos (b t+\phi)+\alpha^{2} b \sin (b t+\phi)\right]}{\left[\lambda_{n}^{4}+\alpha^{4} b^{2}\right]}\right]
\end{gathered}
$$

Where $h_{2}=\frac{\lambda_{n}^{2}}{\alpha^{2}}$

$$
\begin{aligned}
& X_{2}=\frac{A_{0}+g \sin \theta}{\lambda_{n}^{2}}+\frac{A_{1} \lambda_{n}^{2}}{\left(\lambda_{n}^{4}+\alpha^{4}\right)}+\frac{a_{0}\left[\lambda_{n}^{2} \cos \phi+\alpha^{2} b \sin \phi\right]}{\left[\lambda_{n}^{4}+\alpha^{4} b^{2}\right]} \\
& Y=\frac{\pi^{2}}{2} \sum_{n=1}^{\infty} \frac{\lambda_{n}^{2} J_{0}^{2}\left(\lambda_{n}\right)\left\{\begin{array}{r}
h \lambda_{n} u_{2}(h) B_{0}^{\prime}\left(h \lambda_{n}\right)+\frac{1}{\lambda_{n}}\left[A_{0}+A_{1}+a_{0} \cos \phi+g \sin \theta\right] \\
\times\left[h B_{0}^{\prime}\left(h \lambda_{n}\right)-B_{0}^{\prime}\left(h \lambda_{n}\right)\right]
\end{array}\right\}}{\left\{J_{0}^{2}\left(h \lambda_{n}\right)-J_{0}^{2}\left(\lambda_{n}\right)\right\} \lambda_{n}^{2}} \\
& Z=\frac{1}{2}\left[\left\{Y_{0}^{2}\left(h \lambda_{n}\right) J_{1}^{2}\left(\lambda_{n}\right)+J_{0}^{2}\left(h \lambda_{n}\right) Y_{0}^{2}\left(\lambda_{n}\right)+J_{0}^{2}\left(h \lambda_{n}\right) Y_{1}^{2}\left(\lambda_{n}\right)\right\}\right. \\
& -h^{2}\left\{Y_{0}^{2}\left(h \lambda_{n}\right) J_{0}^{2}\left(h \lambda_{n}\right)+Y_{0}^{2}\left(h \lambda_{n}\right) J_{1}^{2}\left(h \lambda_{n}\right)+J_{0}^{2}\left(h \lambda_{n}\right) Y_{0}^{2}\left(\lambda_{n}\right)\right. \\
& \left.\left.+J_{0}^{2}\left(h \lambda_{n}\right) Y_{1}^{2}\left(h \lambda_{n}\right)\right\}\right]-\frac{2 J_{0}^{2}\left(h \lambda_{n}\right) Y_{0}\left(\lambda_{n}\right)}{\left(1-\lambda_{n}\right)}\left[\frac { 1 } { \lambda _ { n } } \left\{Y_{0}\left(\lambda_{n}\right) J_{1}\left(\lambda_{n}\right)\right.\right. \\
& \left.\left.-h Y_{0}\left(h \lambda_{n}\right) J_{1}\left(h \lambda_{n}\right)\right\}-\left\{Y_{0}\left(\lambda_{n}\right) J_{1}\left(\lambda_{n}\right)-h Y_{0}\left(h \lambda_{n}\right) J_{1}\left(h \lambda_{n}\right)\right\}\right]
\end{aligned}
$$

The expression for the flow rate $Q$, which is the volume of the suspension flowing per unit time across a crosssection of the tube, for two phase fluid is given by the equation

$$
Q=Q_{1}+Q_{2}
$$


Where

Then

$$
\begin{gathered}
Q_{1}=2 \pi \int_{0}^{h} r u_{1} d r \text { and } Q_{2}=2 \pi \int_{h}^{1} r u_{2} d r \\
Q_{1}=\frac{2 \pi h J_{1}\left(h \lambda_{n}\right)}{\lambda_{n} J_{0}\left(h \lambda_{n}\right)} u_{1}(h, t), \\
Q_{2}=\frac{2 \pi\left[h B_{0}^{\prime}\left(h \lambda_{n}\right)-B_{0}^{\prime}\left(\lambda_{n}\right)\right]}{\lambda_{n} B_{0}\left(h \lambda_{n}\right)} u_{2}(h, t) .
\end{gathered}
$$

\section{Results And Discussions}

The velocity profile for the effect of permeability of porous medium on pulsatile inclined two layered blood flow with magnetic field and periodic body acceleration is computed by using (21) for different values of permeability of porous medium $K$, Hartmann number $H$, steady state part of pressure gradient $A_{0}$, amplitude of oscillatory part $A_{l}$, amplitude of body acceleration $a_{0}$, gravitational parameter $g$ and inclination angle $\theta$ have been shown through Figures 1- 7.

Figure -1 show that the variation of velocity profile for different values of permeability of porous medium $K$. It can be noted here that as the permeability of porous medium $K$ increases the velocity profile increases. Figure -2 shows that the variation of velocity profile for different values of Hartmann number. It can be noted here that as the Hartmann number $H$ increases the velocity profile decreases. It can easily be seen from figures $3,4 \& 5$ that an increase in the steady state part of pressure gradient $A_{0}$, amplitude of oscillatory part $A_{l}$ and amplitude of body acceleration $a_{0}$ leads to an increase in the velocity profile. From figure -6 it can be observed that the gravitational parameter $g$ increases the velocity profile also increases. From figure -7 it is observed that inclination angle $\theta$ increases the velocity profile also increases.

\section{Conclusion}

Result of the present study suggests that the permeability of porous medium effects the blood velocity of flow. Hartmann number effects largely on the velocity profile of blood flow. From the figure -1 velocity profile is shown. From the figure-2 the amount of deviation in $u(r, \mathrm{t})$ due to $H$ is shown. So, by taking appropriate values of $H$ we may regulate the blood flow velocity. In case of arthritis, gout etc. patients are often advised to take protective pads or tractions. By using an appropriate magnetic field with porous medium it is possible to control blood pressure and also it is effective for conditions such as poor circulation, travel sickness, headaches, pain, muscle sprains, strains and joint pain etc.

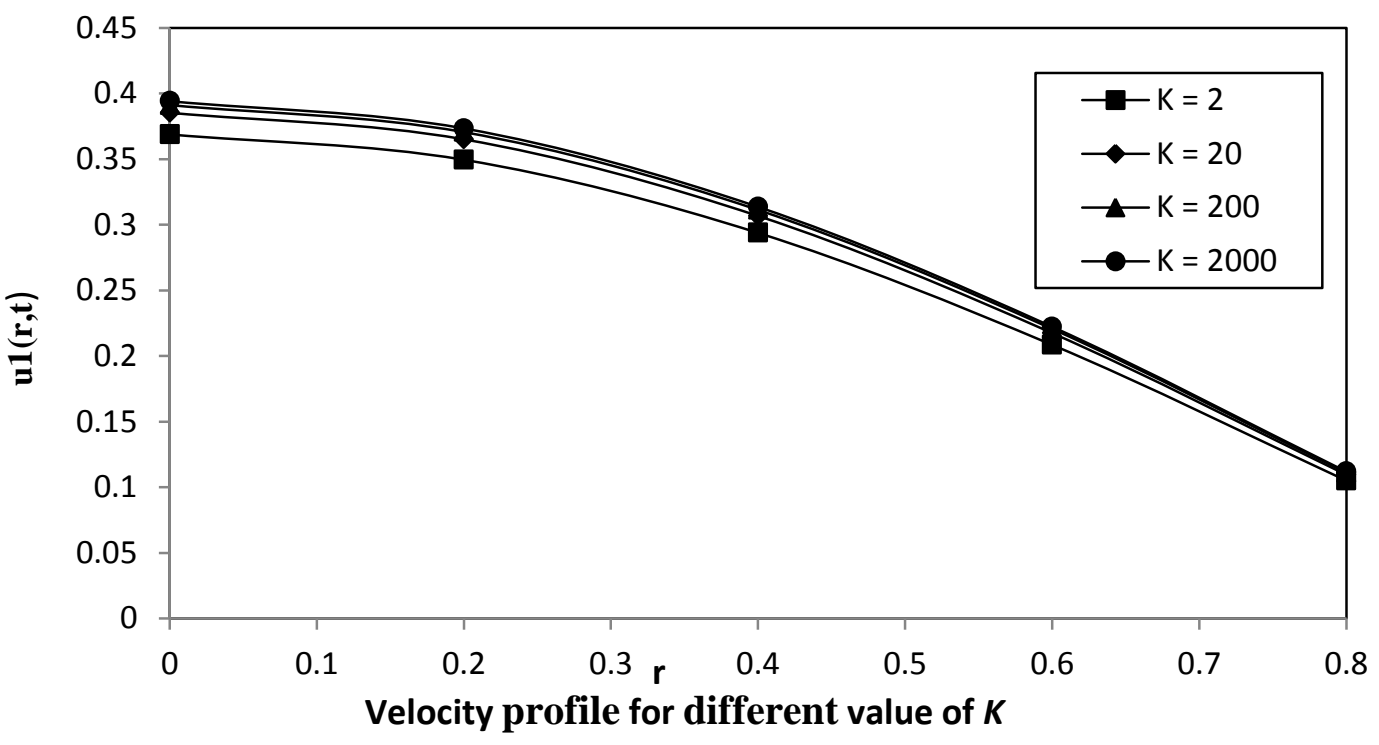

Fig. 1: Variation of velocity profile for different values of $K$;

Taking $\mathrm{A}_{1}=4, \mathrm{a}_{0}=3, \phi=15^{0}, \mathrm{H}=10, \mathrm{~g}=9, \mathrm{t}=0.5, \mathrm{~b}=1, \alpha=1, \bar{\alpha}=1$ 


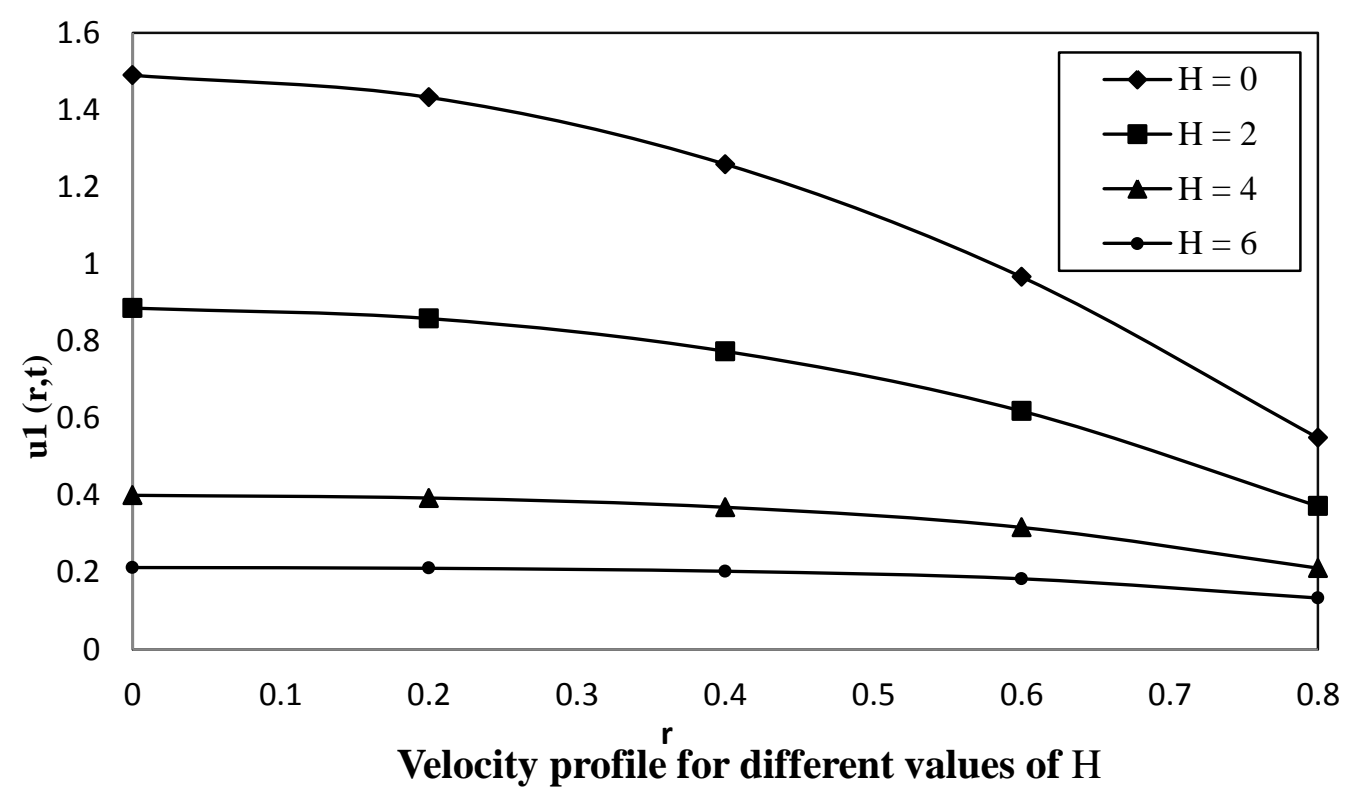

Fig. 2: Variation of velocity profile for different values of $\mathrm{H}$;

Taking $\mathrm{A}_{0}=2, \mathrm{~A}_{1}=4, \mathrm{a}_{0}=3, \phi=15^{0}, \theta=30^{\circ}, \mathrm{t}=0.5, \mathrm{~b}=1, \alpha=1, \bar{\alpha}=1$

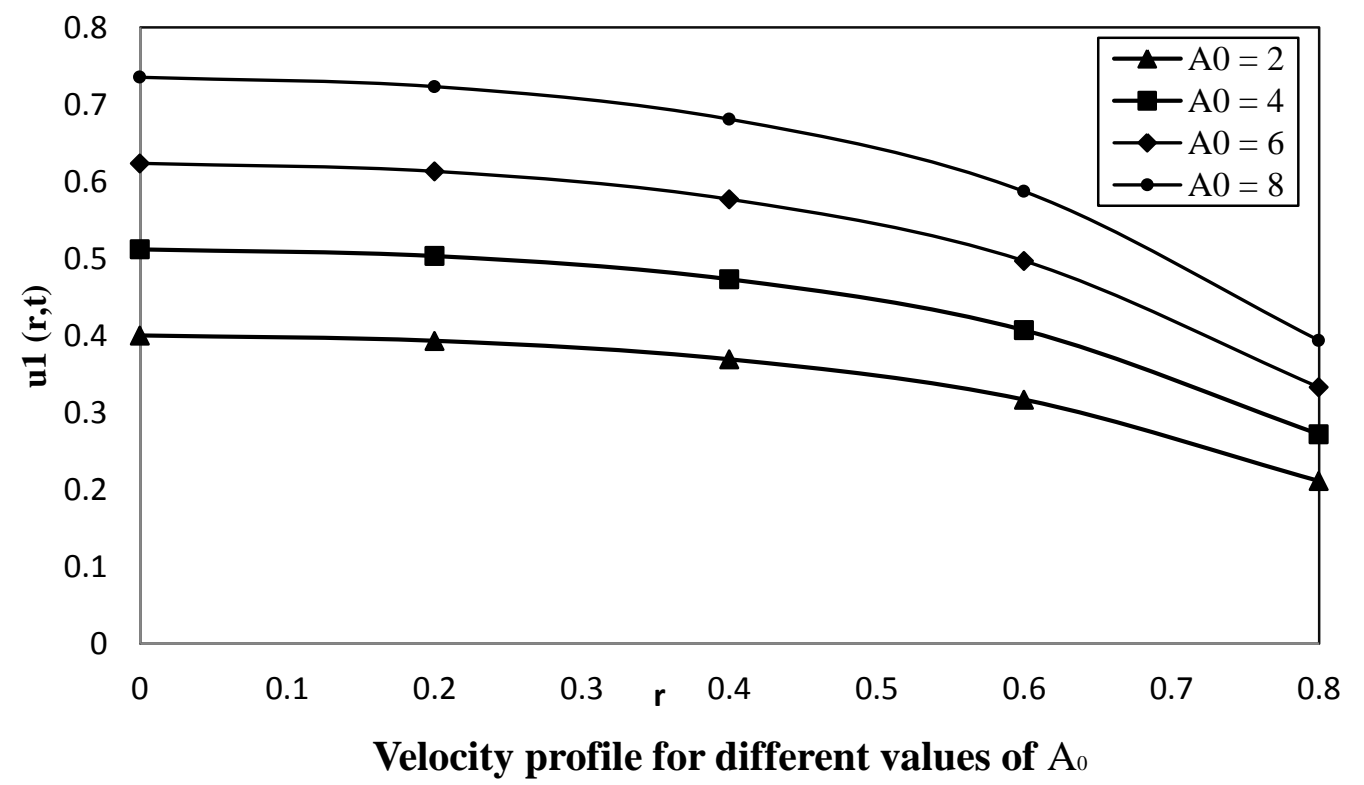

Fig. 3: Variation of velocity profile for different values of $\mathrm{A}_{0}$;

Taking $\mathrm{A}_{1}=4, \mathrm{a}_{0}=3, \phi=15^{0}, \mathrm{H}=10, \mathrm{~g}=9, \mathrm{t}=0.5, \mathrm{~b}=1, \alpha=1, \bar{\alpha}=1$ 


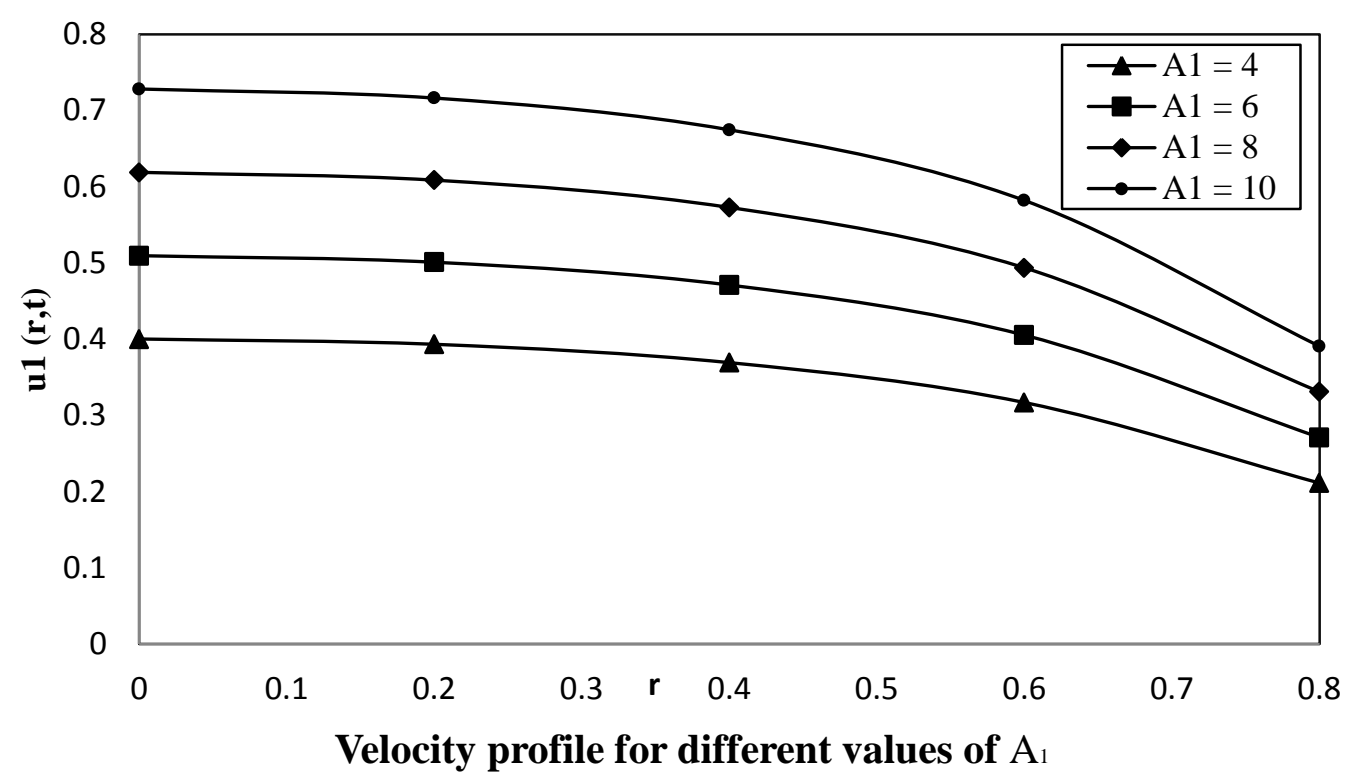

Fig. 4: Variation of velocity profile for different values of $A_{1}$;

Taking $\mathrm{A}_{0}=2, \mathrm{a}_{0}=3, \phi=15^{0}, \mathrm{H}=10, \mathrm{~g}=9, \mathrm{t}=0.5, \mathrm{~b}=1, \alpha=1, \bar{\alpha}=1$

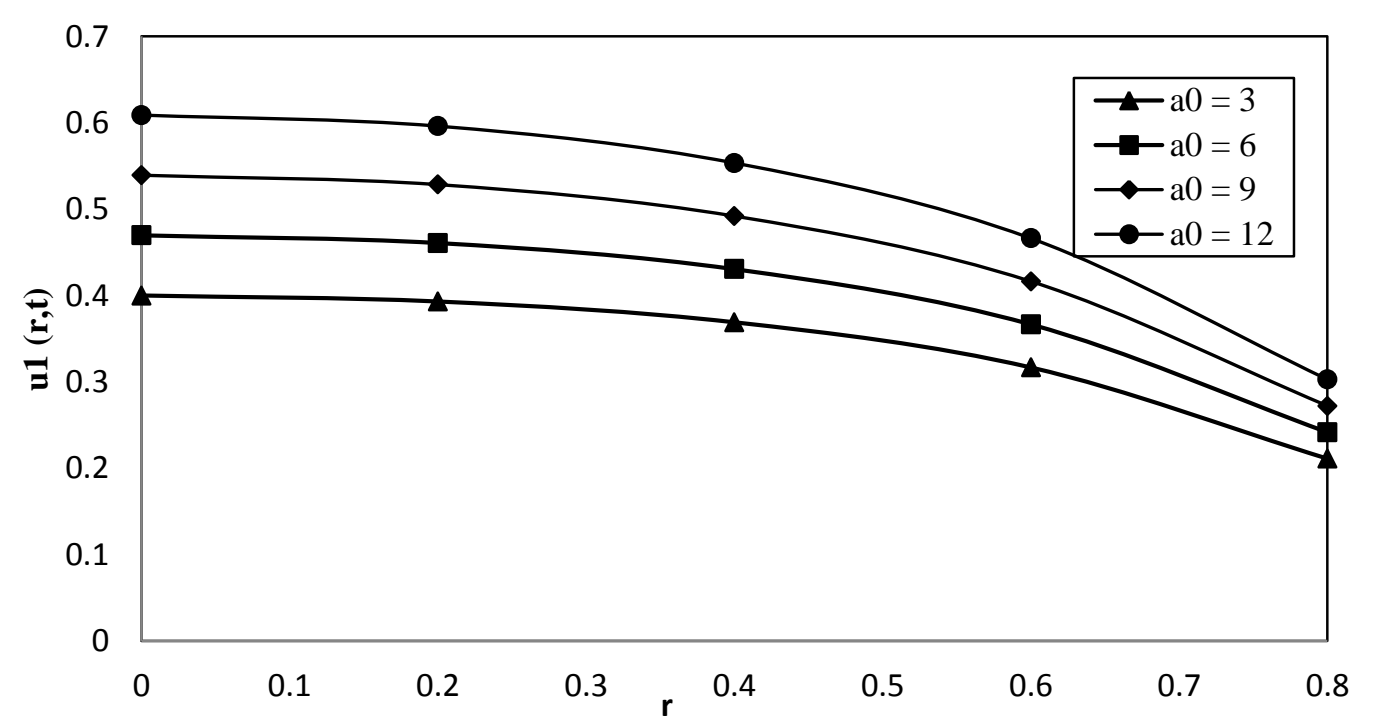

Velocity profile for different values of $\mathrm{a}_{0}$

Fig. 5: Variation of velocity profile for different values of $\mathrm{a}_{0}$;

Taking $\mathrm{A}_{0}=2, \mathrm{~A}_{1}=4, \phi=15^{0}, \mathrm{H}=10, \mathrm{~g}=9, \mathrm{t}=0.5, \mathrm{~b}=1, \alpha=1, \bar{\alpha}=1$ 


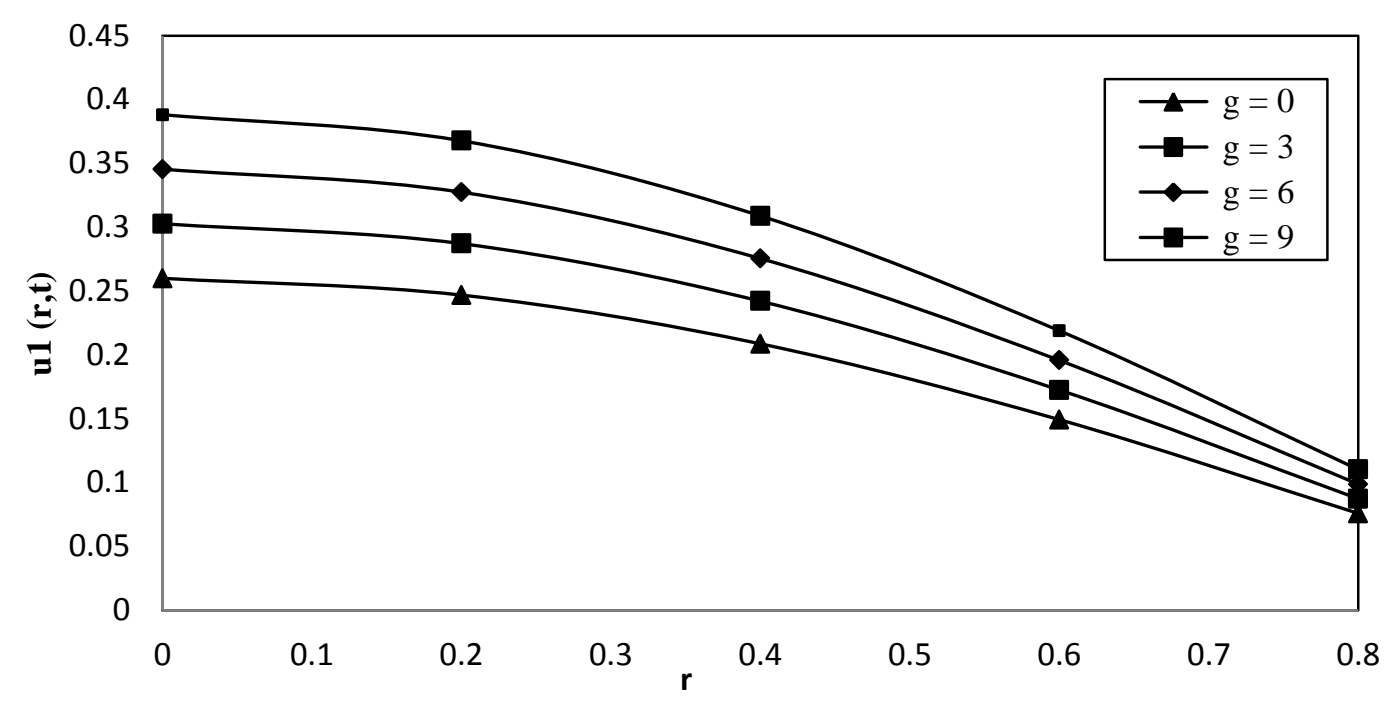

Velocity profile for different values of $\mathrm{g}$

Fig. 6: Variation of velocity profile for different values of $\mathrm{g}$;

Taking $\mathrm{A}_{0}=3, \mathrm{~A}_{1}=4, \mathrm{a}_{0}=3, \phi=15^{0}, \theta=30^{0}, \mathrm{H}=4, \mathrm{t}=0.5, \mathrm{~b}=0.5, \alpha=3, \bar{\alpha}=1$

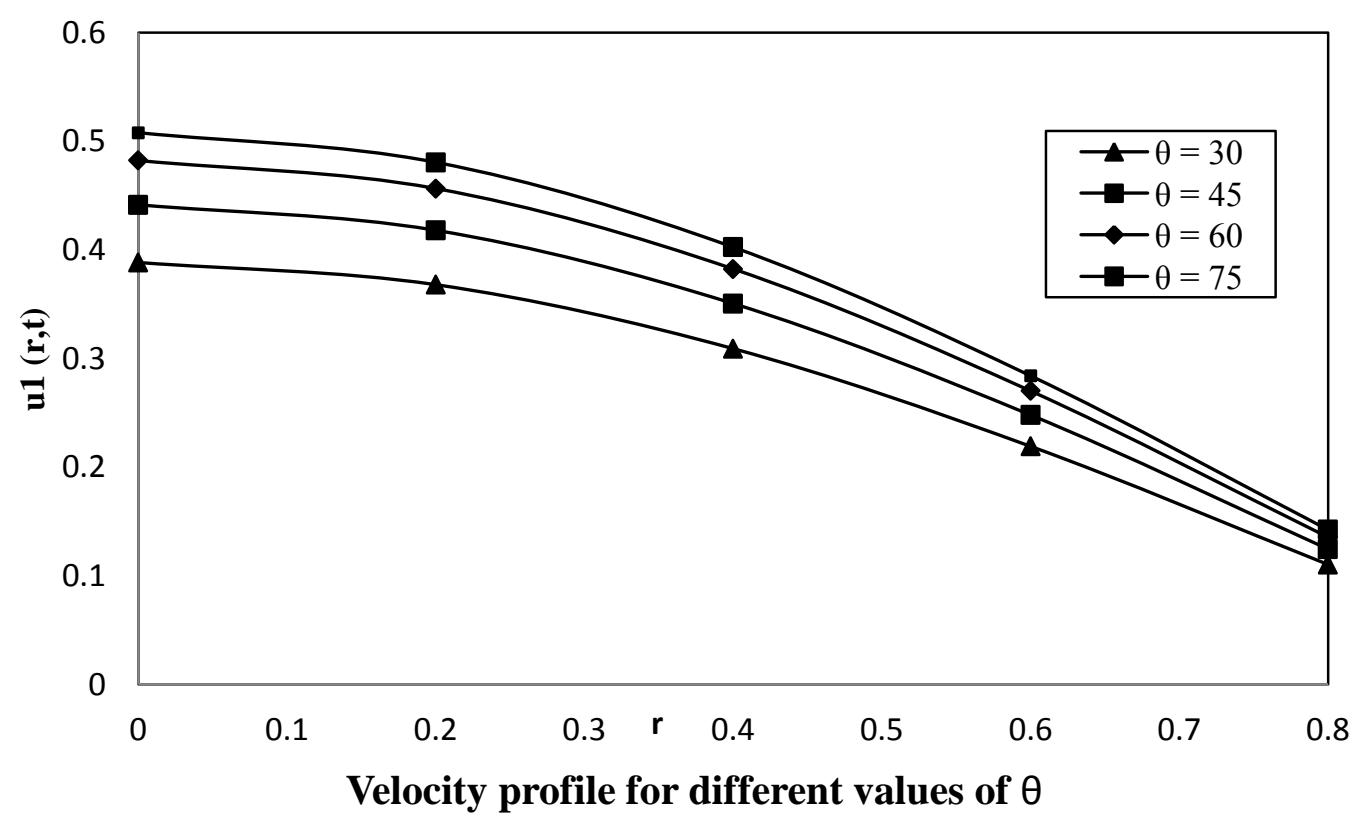

Fig. 7: Variation of velocity profile for different values of $\theta$;

Taking $\mathrm{A}_{0}=3, \mathrm{~A}_{1}=4, \mathrm{a}_{0}=3, \phi=15^{0}, \mathrm{~g}=9, \mathrm{H}=4, \mathrm{t}=0.5, \mathrm{~b}=0.5, \alpha=3, \bar{\alpha}=1$

\section{References}


Agarwal, R. and Varshney.N.K., The effect of magnetic field of pulsatile inclined two layered blood flow with periodic body acceleration, International journal of mechanical Engineering Research. 3(3), 2013, 187-196 .

[2] Agarwal, R. and Varshney, N. K., Inclined arterial MHD pulsatile blood flow through a porous medium with periodic body acceleration, Jour. PAS, Mathematical Science. 17, 2011, 304-313.

[3] Amos, E. and Ogulu, A.,Magnetic effect on pulsatile flow in a constricted axisymmetric tube, Indian J. Pure and appl. Math. 34(9), 2003, 1315-1326.

[4] Astarita, G., Marrucii and Palumbo,G., Non-Newtonian gravity flow on inclined surface, Ind. Engg. Chem. Fundam. 3, $1964,333$.

[5] Chaturani, P. and Palanisamy, V., Casson fluid model for pulsatile flow of blood under periodic body acceleration, Biorheology, 27, 1990, 619-630.

[6] Chaturani, P. and Palanisamy V., Pulsatile flow of Power-law fluid model for blood flow under periodic body acceleration, Biorheology. 27, 1990, 747-758.

[7] Chaturani, P and Wassf Isaac, A. S. A., blood flow with body acceleration forces, Int. J. Engg. Sci. 33(12), 1995, 1807-1820.

[8] El-Shahed,M., Pulsatile flow of blood through a stenosed porous medium under periodic body acceleration, Appl. Math. Comput, $138(2), 2003,479-488$.

[9] Haldar, K and Ghosh SN., Effect of magnetic field on blood flow through an indented tube in the presence of erythrocytes, Indian. J. Pure appl. Math, 25(3), 1994, 345-352.

[10] Kavitha A, Hemadri Reddy R, Sreenadh S, Saravana R and Srinivas A.N.S, Flow of a micropolar fluid in a vertical channel with long wave length approximation, Advances in Applied Science Research, 2(1), 2011, $269-279$.

[11] Rathod V. P. and Habeeb Ahmed, Pulsatile flow of blood through a stenosed inclined tube under periodic body acceleration, Journal of Indian Acad. Math. 2(1), 2008, 137-151.

[12] Rathod V. P. and HosurkerShrikanth, G., Pulsatile two layered flow under periodic body acceleration, Bull. Of Pure and Appl. Sci., 17(1), 1998, 125-134.

[13] Rathod, V. P. and Channakota,M,M., A study ureteral peristalsis in cylindrical tube through porous medium, Advances in Applied Science Research, 2(3), 2011, 134-140.

[14] Rathod, V. P. and Thippeswamy, G., MHD flow of Rivlin-Ericksen fluid through an inclined channel J. The Math.Edu. XXXIII(1), 1999, 40-49.

[15] Ravi Kumar Y.V.K, Krishna Kumari P, A numerical model for the effect of stenosis shape on blood flow through an artery using power-law fluid Peristaltic transport of a power-law fluid in an asymmetric channel bounded by permeable walls, Advances in Applied Science Research 2(3), 2011, 396-406.

Singh,S., and Shah, Ratan,R., A numerical model for the effect of stenosis shape on blood flow through an artery using power-law fluid, Advances in Applied Science Research, 1(1),2010, 66-73.

[17] Srivastava, V.P. and Saxena, M., Two-layered model of Casson's fluid flow through stenotic blood vessels application to cardiovascular system, J Biomech. 27, 1994, 921-928.

[18] S.V.H.N. Krishna Kumari P, Ramana Murthy M.V, ChennaKesava Reddy M and Ravi Kumar Y.V.K, Peristaltic pumping of a magnetohydrodynamic casson fluid in an inclined channel, Advances in Applied Science Research 2(2), 2011, 428-436. Stokes, V. K., Couple-stress in fluid, The Physics of fluid, 9, 1966, 1709-1715.

[20] Sud, V.K. and Sekhon, G.S., Blood flow through the human arterial system in the presence of a steady magnetic field, Phys. Med. Biol, 34, 1989, 36-42.

[21] Sud, V.K., Suri, P.K. and Mishra, R.K., Effect of magnetic field on oscillating blood in arteries, Studia Biophysica,46, 1974, 163169.

[22] Tranter, Wrokshop, B.L., Integral transform in mathematical physics, infinite transform, London,(1956). 88(2). 